\title{
A Schwartz type algebra for the Tangent groupoid
}

\author{
Paulo Carrillo Rouse
}

\begin{abstract}
We construct an algebra of smooth functions over the tangent groupoid associated to any Lie groupoid. This algebra is a field of algebras over the closed interval $[0,1]$ which fiber at zero is the algebra of Schwartz functions over the Lie algebroid, whereas any fiber out of zero is the convolution algebra of the initial groupoid. Our motivation comes from index theory for Lie groupoids. In fact, our construction gives an intermediate algebra between the enveloping $C^{*}$-algebra and the convolution algebra of compactly supported functions of the tangent groupoid; and it will allows us, in a further work, to define other analytic index morphisms as a sort of "deformations".
\end{abstract}

Mathematics Subject Classification (2000).

Primary 58-06, 19-06; Secondary 58H15, 19K56.

Keywords.

Lie groupoids, Tangent groupoid, K-theory, Index theory.

\section{Introduction}

The concept of groupoid is central in non commutative geometry. Groupoids generalize the concepts of spaces, groups and equivalence relations. It is clear nowadays that groupoids are natural substitutes of singular spaces. Many people have contributed to realizing this idea. We can find for instance a groupoid-like treatment in Dixmier's works on transformation groups, 11, or in Brown-Green-Rieffel's work on orbit classification of relations, 3 . In foliation theory, several models for the leaf space of a foliation were realized using groupoids, mainly by people like Haefliger ([12]) and Wilkelnkemper ([24]), for mention some of them. There is also the case of Orbifolds, these can be seen indeed as étale groupoids, (see for example Moerdijk's paper [17]). There are also some particular groupoid models for manifolds with corners and conic manifoldss worked by people like Monthubert [18, Debord-Lescure-Nistor ([10]) and Aastrup-Melo-Monthubert-Schrohe ([1]) for example.

The way we treat "singular spaces" in non commutative geometry is by associating to them algebras. In the case when the "singular space" is represented by a Lie groupoid, we can, for instance, consider the convolution algebra of differentiable functions with compact support over the groupoid (see Connes or Paterson's books [8] and 22]). This last algebra plays the role of the algebra of smooth func- 
tions over the "singular space" represented by the groupoid. From the convolution algebra it is also possible to construct a $C^{*}$-algebra, $C^{*}(\mathscr{G})$, that plays, in some sense, the role of the algebra of continuous functions over the "singular space". The idea of associating algebras in this sense can be traced back in works of Dixmier ([1]) for transformation groups, Connes ([6]) for foliations and Renault ([23]) for locally compact groupoids, for mention some of them.

Using methods of Noncommutative Geometry, we would like to get invariants of this algebras, and hence, of the spaces they represent. For that, Connes showed that many groupoids and algebras associated to them appeared as 'non commutative analogues' of spaces to which many tools of geometry (and topology) such as K-theory and Characteristic classes could be applied ([7, [8]). One classical way to obtain invariants in classical geometry (topology), is through the index theory in the sense of Atiyah-Singer. In the Lie groupoid case, there is a Pseudodifferential calculus, developed by Connes ([6]), Monthubert-Pierrot ([19]) and Nistor-Weinstein-Xu (21]) in general. Some interesting particular cases were treated in the groupoid-spirit by Melrose ([16]), Moroianu ([20]) and others (see [1]). Let $\mathscr{G} \rightrightarrows \mathscr{G}^{(0)}$ be a Lie groupoid, there is an analytic index morphism, (see [19]),

$$
\operatorname{ind}_{a}: K^{0}\left(A^{*} \mathscr{G}\right) \rightarrow K_{0}\left(C^{*}(\mathscr{G})\right)
$$

where $A \mathscr{G}$ is the Lie algebroid of $\mathscr{G}$. The " $C^{*}$-index" $i n d_{a}$ is a homotopy invariant of the $\mathscr{G}$-pseudodifferential elliptic operators and has proved to be very useful in very different situations, (see [2, 9], [10, for example). One way to define the above index map is using the Connes' tangent groupoid associated to $\mathscr{G}$ as explained by Hilsum and Skandalis in [13] or by Monthubert and Pierrot in [19]. The tangent groupoid is a Lie groupoid

$$
\mathscr{G}^{T} \rightrightarrows \mathscr{G}^{(0)} \times[0,1]
$$

with $\mathscr{G}^{T}:=A \mathscr{G} \times\{0\} \bigsqcup \mathscr{G} \times(0,1]$ and the groupoid structure is given by the groupoid structure of $A \mathscr{G}$ at $t=0$ and by the groupoid structure of $\mathscr{G}$ for $t \neq 0$. One of the main features about the tangent groupoid is that its $C^{*}$-algebra $C^{*}\left(\mathscr{G}^{T}\right)$ is a continuous field of $C^{*}$-algebras over the closed interval $[0,1]$, with associated fiber algebras

$$
\begin{gathered}
C_{0}\left(A^{*} \mathscr{G}\right) \text { at } t=0, \text { and } \\
C^{*}(\mathscr{G}) \text { for } t \neq 0 .
\end{gathered}
$$

In fact, it gives a $C^{*}$-algebraic quantization of the Poisson manifold $A^{*} \mathscr{G}$ (in the sense of [14]), and this is the main point why it allows to define the index morphism as a sort of "deformation". Thus, the tangent groupoid construction has been very useful in index theory $([2,[10,[13])$ but also for other purposes ([14, [21]).

Now, to understand the purpose of the present work, let us first say that the indices (in the sense of Atiyah-Singer-Connes) have not necessarily to be considered as elements in $K_{0}\left(C^{*}(\mathscr{G})\right)$. Indeed, it is possible to consider indices in $K_{0}\left(C_{c}^{\infty}(\mathscr{G})\right)$. The $C_{c}^{\infty}$-indices are more refined but they have several inconvenients (see Alain Connes' book section 9. $\beta$ for a discussion on this matter), nevertheless this kind 
of indices have the great advantage that one can apply to them the existent tools (such as pairings with cyclic cocycles or Chern-Connes character) in order to obtain numerical invariants.

In this work we begin a study of more refined indices. In particular we are looking for indices between the $C_{c}^{\infty}$ and the $C^{*}$-levels; trying to keep the advantages of both approaches (see [5] for a more complete discussion). In the case of Lie groupoids this refinement could mean forget for a moment the powerful tools of the theory of $C^{*}$-algebras and instead, working in a purely algebraic and geometric level. In the present article, we construct an algebra of $C^{\infty}$ functions over $\mathscr{G}^{T}$, denoted by $\mathscr{S}_{r, c}\left(\mathscr{G}^{T}\right)$. This algebra is also a field of algebras over the closed interval $[0,1]$, with associated fiber algebras,

$$
\begin{gathered}
\mathscr{S}(A \mathscr{G}), \text { at } t=0, \text { and } \\
C_{c}^{\infty}(\mathscr{G}) \text { for } t \neq 0,
\end{gathered}
$$

where $\mathscr{S}(A \mathscr{G})$ is the Schwartz algebra of the Lie algebroid. Furthermore, we will have

$$
C_{c}^{\infty}\left(\mathscr{G}^{T}\right) \subset \mathscr{S}_{r, c}\left(\mathscr{G}^{T}\right) \subset C^{*}\left(\mathscr{G}^{T}\right),
$$

as inclusions of algebras. Let us explain in some words why we define an algebra over the tangent groupoid such that in zero it is Schwartz: The "Schwartz algebras" have in general the good $K$-theory groups. For example, we are interested in the symbols of $\mathscr{G}$-PDO and more precisely in their homotopy classes in $K$-theory, that is, we are interested in the group $K^{0}\left(A^{*} \mathscr{G}\right)=K_{0}\left(C_{0}\left(A^{*} \mathscr{G}\right)\right)$. Here it would not be enough to take the $K$-theory of $C_{c}^{\infty}(A \mathscr{G})$ (see the example in 8 p.142), however it is enough to consider the Schwartz algebra $\mathscr{S}\left(A^{*} \mathscr{G}\right)$. Indeed, the Fourier transform shows that this last algebra is stable under holomorphic calculus on $C_{0}\left(A^{*} \mathscr{G}\right)$ and so it has the "good" $K$-theory, meaning that $K^{0}\left(A^{*} \mathscr{G}\right)=K_{0}\left(\mathscr{S}\left(A^{*} \mathscr{G}\right)\right)$. None of the inclusions in (11) is stable under holomorphic calculus, but that is precisely what we wanted because our algebra $\mathscr{S}_{r, c}\left(\mathscr{G}^{T}\right)$ have the remarkable property that its evaluation at zero is stable under holomorphic calculus while its evaluation at one (for example) is not.

The algebra $\mathscr{S}_{r, c}\left(\mathscr{G}^{T}\right)$ is, as vector space, a particular case of a more general construction that we do for "Deformation to the normal cone manifolds" from which the tangent groupoid is a special case (see [4] and [13]). A deformation to the normal cone manifold (DNC for simplify) is a manifold associated to an injective immersion $X \hookrightarrow M$ that is considered as a sort of blow up in differential geometry. The construction of a DNC manifold has very nice functorial properties (section 3) which we exploit to achieve our construction. We think that our construction could be used also for other purposes, for example, it seems that it could help to give more understanding in quantization theory (see again [4]).

The article is organized as follows. In the second section we recall the basic facts about Lie groupoids. We explain very briefly how to define the convolution algebra $C_{c}^{\infty}(\mathscr{G})$. In the third section we explain the "deformation to the normal cone" construction associated to an injective immersion. Even if this could be considered as classical material, we do it in some detail since we will use in the 
sequel very explicit descriptions that we could not find elsewhere. We also review some functorial properties associated to these deformations. A particular case of this construction is the tangent groupoid associated to a Lie groupoid. In the fourth section we start by constructing a vector space $\mathscr{S}_{r, c}\left(\mathscr{D}_{X}^{M}\right)$ for any Deformation to the normal cone manifold $\mathscr{D}_{X}^{M}$; this space already exhibits the characteristic of being a field of vector spaces over the closed interval $[0,1]$, such that in zero we have a Schwartz space while out of zero we have $C_{c}^{\infty}(M)$. We then define the algebra $\mathscr{S}_{r, c}\left(\mathscr{G}^{T}\right)$, the main result is precisely that the product is well defined. The last section is devoted to motivate the construction of our algebra by explaining in a few words some further developements that will immediately follow from this work. All the results of the present work are part of the author's PHD thesis.

I want to thank my PHD advisor, Georges Skandalis, for all the ideas that he shared with me. I would also like to thank him for all the comments and remarks he made to the present work. I would also like to thank the referee for the useful comments he made for improving this paper.

\section{Lie groupoids}

Let us recall what a groupoid is:

Definition 2.1. A groupoid consists of the following data: two sets $\mathscr{G}$ and $\mathscr{G}^{(0)}$, and maps

. $s, r: \mathscr{G} \rightarrow \mathscr{G}^{(0)}$ called the source and target map respectively,

- $m: \mathscr{G}^{(2)} \rightarrow \mathscr{G}$ called the product map (where $\mathscr{G}^{(2)}=\{(\gamma, \eta) \in \mathscr{G} \times \mathscr{G}: s(\gamma)=$ $r(\eta)\})$,

$\cdot u: \mathscr{G}^{(0)} \rightarrow \mathscr{G}$ the unit map and

. $i: \mathscr{G} \rightarrow \mathscr{G}$ the inverse map

such that, if we note $m(\gamma, \eta)=\gamma \cdot \eta, u(x)=x$ and $i(\gamma)=\gamma^{-1}$, we have

1. $\gamma \cdot(\eta \cdot \delta)=(\gamma \cdot \eta) \cdot \delta, \forall \gamma, \eta, \delta \in \mathscr{G}$ when this is possible.

2. $\gamma \cdot x=\gamma$ and $x \cdot \eta=\eta, \forall \gamma, \eta \in \mathscr{G}$ with $s(\gamma)=x$ and $r(\eta)=x$.

3. $\gamma \cdot \gamma^{-1}=u(r(\gamma))$ and $\gamma^{-1} \cdot \gamma=u(s(\gamma)), \forall \gamma \in \mathscr{G}$.

4. $r(\gamma \cdot \eta)=r(\gamma)$ and $s(\gamma \cdot \eta)=s(\eta)$.

Generally, we denote a groupoid by $\mathscr{G} \rightrightarrows \mathscr{G}^{(0)}$ where the parallel arrows are the source and target maps and the other maps are given.

Now, a Lie groupoid is a groupoid in which every set and map appearing in the last definition is $C^{\infty}$ (possibly with borders), and the source and target maps are submersions. For $A, B$ subsets of $\mathscr{G}^{(0)}$ we use the notation $\mathscr{G}_{A}^{B}$ for the subset $\{\gamma \in \mathscr{G}: s(\gamma) \in A, r(\gamma) \in B\}$.

All along this paper, $\mathscr{G} \rightrightarrows \mathscr{G}^{(0)}$ is going to be a Lie groupoid. We recall how to define an algebra structure in $C_{c}^{\infty}(\mathscr{G})$ using smooth Haar systems. 
Definition 2.2. A smooth Haar system over a Lie groupoid consists of a family of measures $\mu_{x}$ in $\mathscr{G}_{x}$ for each $x \in \mathscr{G}^{(0)}$ such that,

- for $\eta \in \mathscr{G}_{x}^{y}$ we have the following compatibility condition:

$$
\int_{\mathscr{G}_{x}} f(\gamma) d \mu_{x}(\gamma)=\int_{\mathscr{G}_{y}} f(\gamma \circ \eta) d \mu_{y}(\gamma)
$$

- for each $f \in C_{c}^{\infty}(\mathscr{G})$ the map

$$
x \mapsto \int_{\mathscr{G}_{x}} f(\gamma) d \mu_{x}(\gamma)
$$

belongs to $C_{c}^{\infty}\left(\mathscr{G}^{(0)}\right)$

A Lie groupoid always posses a smooth Haar system. In fact, if we fix a smooth (positive) section of the 1-density bundle associated to the Lie algebroid we obtain a smooth Haar system in a canonical way. The advantage of using 1-densities is that the measures are locally equivalent to the Lebesgue measure. We suppose for the rest of the paper a given smooth Haar system given by 1-densities (for complete details see [22]). We can now define a convolution product on $C_{c}^{\infty}(\mathscr{G})$ : Let $f, g \in C_{c}^{\infty}(\mathscr{G})$, we set

$$
(f * g)(\gamma)=\int_{\mathscr{G}_{s(\gamma)}} f\left(\gamma \cdot \eta^{-1}\right) g(\eta) d \mu_{s(\gamma)}(\eta)
$$

This gives a well defined associative product.

Remark 2.3. There is a way to avoid the Haar system when one works with Lie groupoids, using half densities (see Connes' book [8]).

\section{Deformation to the normal cone}

Let $M$ be a $C^{\infty}$ manifold and $X \subset M$ be a $C^{\infty}$ submanifold. We denote by $\mathscr{N}_{X}^{M}$ the normal bundle to $X$ in $M$, i.e., $\mathscr{N}_{X}^{M}:=T_{X} M / T X$. We define the following set

$$
\mathscr{D}_{X}^{M}:=\mathscr{N}_{X}^{M} \times 0 \bigsqcup M \times(0,1]
$$

The purpose of this section is to recall how to define a $C^{\infty}$-structure with boundary in $\mathscr{D}_{X}^{M}$. This is more or less classical, for example it was extensively used in [13]. Here we are only going to do a sketch.

Let us first consider the case where $M=\mathbf{R}^{n}$ and $X=\mathbf{R}^{p} \times\{0\}$ (where we identify canonically $\left.X=\mathbf{R}^{p}\right)$. We denote by $q=n-p$ and by $\mathscr{D}_{p}^{n}$ for $\mathscr{D}_{\mathbf{R}^{p}}^{\mathbf{R}^{n}}$ as above. In this case we clearly have that $\mathscr{D}_{p}^{n}=\mathbf{R}^{p} \times \mathbf{R}^{q} \times[0,1]$ (as a set). Consider the bijection

$$
\Psi: \mathbf{R}^{p} \times \mathbf{R}^{q} \times[0,1] \rightarrow \mathscr{D}_{p}^{n}
$$


given by

$$
\Psi(x, \xi, t)= \begin{cases}(x, \xi, 0) & \text { if } t=0 \\ (x, t \xi, t) & \text { if } t>0\end{cases}
$$

which inverse is given explicitly by

$$
\Psi^{-1}(x, \xi, t)=\left\{\begin{array}{cc}
(x, \xi, 0) & \text { if } t=0 \\
\left(x, \frac{1}{t} \xi, t\right) & \text { if } t>0
\end{array}\right.
$$

We can consider the $C^{\infty}$-structure with border on $\mathscr{D}_{p}^{n}$ induced by this bijection.

In the general case. Let $(\mathscr{U}, \phi)$ be a local chart in $M$ and suppose it is an $X$-slice, so that it satisfies

1) $\phi: \mathscr{U} \stackrel{\cong}{\rightrightarrows} U \subset \mathbf{R}^{p} \times \mathbf{R}^{q}$

2) If $\mathscr{U} \cap X=\mathscr{V}, \mathscr{V}=\phi^{-1}\left(U \cap \mathbf{R}^{p} \times\{0\}\right)$ (we note $\left.V=U \cap \mathbf{R}^{p} \times\{0\}\right)$

With this notation we have that $\mathscr{D}_{V}^{U} \subset \mathscr{D}_{p}^{n}$ is an open subset. We may define a function

$$
\tilde{\phi}: \mathscr{D}_{\mathscr{V}}^{\mathscr{U}} \rightarrow \mathscr{D}_{V}^{U}
$$

in the following way: For $x \in \mathscr{V}$ we have $\phi(x) \in \mathbf{R}^{p} \times\{0\}$. If we write $\phi(x)=$ $\left(\phi_{1}(x), 0\right)$, then

$$
\phi_{1}: \mathscr{V} \rightarrow V \subset \mathbf{R}^{p}
$$

is a diffeomorphism, where $V=U \cap\left(\mathbf{R}^{p} \times\{0\}\right)$. We set $\tilde{\phi}(v, \xi, 0)=\left(\phi_{1}(v), d_{N} \phi_{v}(\xi), 0\right)$ and $\tilde{\phi}(u, t)=(\phi(u), t)$ for $t \neq 0$. Here $d_{N} \phi_{v}: \mathscr{N}_{v} \rightarrow \mathbf{R}^{q}$ is the normal component of the derivate $d \phi_{v}$ for $v \in \mathscr{V}$. It is clear that $\tilde{\phi}$ is also a bijection (in particular it induces a $C^{\infty}$ structure with border over $\mathscr{D}_{\mathscr{V}}^{\mathscr{U}}$ ).

Let us define, with the same notations as above, the following set

$$
\Omega_{V}^{U}=\left\{(x, \xi, t) \in \mathbf{R}^{p} \times \mathbf{R}^{q} \times[0,1]:(x, t \cdot \xi) \in U\right\} .
$$

which is an open subset of $\mathbf{R}^{p} \times \mathbf{R}^{q} \times[0,1]$ and thus a $C^{\infty}$ manifold (with border). It is immediate that $\mathscr{D}_{V}^{U}$ is diffeomorphic to $\Omega_{V}^{U}$ through the restriction of $\Psi$, used in (3). Now we consider an atlas $\left\{\left(\mathscr{U}_{\alpha}, \phi_{\alpha}\right)\right\}_{\alpha \in \Delta}$ of $M$ consisting of $X$-slices. It is clear that

$$
\mathscr{D}_{X}^{M}=\cup_{\alpha \in \Delta} \mathscr{D}_{\mathscr{V}_{\alpha}}^{\mathscr{U}_{\alpha}}
$$

and if we take $\mathscr{D}_{\mathscr{V}_{\alpha}}^{\mathscr{U}_{\alpha}} \stackrel{\varphi_{\alpha}}{\rightarrow} \Omega_{V_{\alpha}}^{U_{\alpha}}$ defined as the composition

$$
\mathscr{D}_{\mathscr{V}_{\alpha}}^{\mathscr{U}_{\alpha}} \stackrel{\phi_{\alpha}}{\rightarrow} \mathscr{D}_{V_{\alpha}}^{U_{\alpha}} \stackrel{\Psi_{\alpha}^{-1}}{\rightarrow} \Omega_{V_{\alpha}}^{U_{\alpha}}
$$

then we obtain the following result.

Proposition 3.1. $\left\{\left(\mathscr{D}_{\mathscr{V}_{\alpha}}^{\mathscr{U}_{\alpha}}, \varphi_{\alpha}\right)\right\}_{\alpha \in \Delta}$ is a $C^{\infty}$ atlas with border over $\mathscr{D}_{X}^{M}$.

In fact the proposition can be proved directly from the following elementary lemma 
Lemma 3.2. Let $F: U \rightarrow U^{\prime}$ a $C^{\infty}$ diffeomorphism where $U \subset \mathbf{R}^{p} \times \mathbf{R}^{q}$ and $U^{\prime} \subset \mathbf{R}^{p} \times \mathbf{R}^{q}$ are open subsets. We write $F=\left(F_{1}, F_{2}\right)$ and we suppose that $F_{2}(x, 0)=0$. Then the function $\tilde{F}: \Omega_{V}^{U} \rightarrow \Omega_{V^{\prime}}^{U^{\prime}}$ defined by

$$
\tilde{F}(x, \xi, t)=\left\{\begin{array}{cc}
\left(F_{1}(x, 0), \frac{\partial F_{2}}{\partial \xi}(x, 0) \cdot \xi, 0\right) & \text { if } t=0 \\
\left(F_{1}(x, t \xi), \frac{1}{t} F_{2}(x, t \xi), t\right) & \text { if } t>0
\end{array}\right.
$$

is a $C^{\infty}$ map.

Proof. Since the result will hold if and only if it is true in each coordinate, it is enough to prove that if we have $F: U \rightarrow \mathbf{R}$ a $C^{\infty}$ map with $F(x, 0)=0$, then the $\operatorname{map} \tilde{F}: \Omega_{V}^{U} \rightarrow \mathbf{R}$ given by

$$
\tilde{F}(x, \xi, t)=\left\{\begin{array}{cc}
\frac{\partial F}{\partial \xi}(x, 0) \cdot \xi & \text { if } t=0 \\
\frac{1}{t} F(x, t \xi) & \text { if } t>0
\end{array}\right.
$$

is a $C^{\infty}$ map. For that, we write

$$
F(x, \xi)=\frac{\partial F}{\partial \xi}(x, 0) \cdot \xi+h(x, \xi) \cdot \xi
$$

with $h: U \rightarrow \mathbf{R}^{q}$ a $C^{\infty}$ map such that $h(x, 0)=0$. Then

$$
\frac{1}{t} F(x, t \xi)=\frac{\partial F}{\partial \xi}(x, 0) \cdot \xi+h(x, t \xi) \cdot \xi
$$

from which we immediately get the result.

Definition 3.3 (DNC). Let $X \subset M$ be as above. The set $\mathscr{D}_{X}^{M}$ provided with the $C^{\infty}$ structure with border induced by the atlas described in the last proposition is called 'The deformation to normal cone associated to $X \subset M^{\prime}$. We will often write DNC instead of Deformation to the normal cone.

Remark 3.4. Following the same steps, it is possible to define a deformation to the normal cone associated to an injective immersion $X \hookrightarrow M$.

Examples 3.5. Let us mention some basic examples of DCN manifolds $\mathscr{D}_{X}^{M}$ :

1. Consider the case when $X=\emptyset$. We have that $\mathscr{D}_{\emptyset}^{M}=M \times(0,1]$ with the usual $C^{\infty}$ structure on $M \times(0,1]$. We used this fact implicitly for cover $\mathscr{D}_{X}^{M}$ as in (41).

2. Consider the case when $X \subset M$ is an open subset. Then we do not have any deformation at zero and we immediately see by definition that $\mathscr{D}_{X}^{M}$ is just the open subset of $M \times[0,1]$ consisting in the union of $X \times[0,1]$ and $M \times(0,1]$.

The most important feature about the DNC construction is that it is in some sense functorial. More explicitly, let $(M, X)$ and $\left(M^{\prime}, X^{\prime}\right)$ be $C^{\infty}$-couples as above and let $F:(M, X) \rightarrow\left(M^{\prime}, X^{\prime}\right)$ be a couple morphism, i.e., a $C^{\infty} \operatorname{map} F: M \rightarrow M^{\prime}$, 
with $F(X) \subset X^{\prime}$. We define $\mathscr{D}(F): \mathscr{D}_{X}^{M} \rightarrow \mathscr{D}_{X^{\prime}}^{M^{\prime}}$ by the following formulas:

$$
\begin{aligned}
& \mathscr{D}(F)(x, \xi, 0)=\left(F(x), d_{N} F_{x}(\xi), 0\right) \text { and } \\
& \mathscr{D}(F)(m, t)=(F(m), t) \text { for } t \neq 0,
\end{aligned}
$$

where $d_{N} F_{x}$ is by definition the map

$$
\left(\mathscr{N}_{X}^{M}\right)_{x} \stackrel{d_{N} F_{x}}{\longrightarrow}\left(\mathscr{N}_{X^{\prime}}^{M^{\prime}}\right)_{F(x)}
$$

induced by $T_{x} M \stackrel{d F_{x}}{\longrightarrow} T_{F(x)} M^{\prime}$.

We have the following proposition, which is also an immediate consequence of the lemma above.

Proposition 3.6. The map $\mathscr{D}(F): \mathscr{D}_{X}^{M} \rightarrow \mathscr{D}_{X^{\prime}}^{M^{\prime}}$ is $C^{\infty}$.

Remark 3.7. If we consider the category $\mathscr{C}_{2}^{\infty}$ of $C^{\infty}$ pairs given by a $C^{\infty}$ manifold and a $C^{\infty}$ submanifold and pair morphisms as above, we can reformulate the proposition and say that we have a functor

$$
\mathscr{D}: \mathscr{C}_{2}^{\infty} \rightarrow \mathscr{C}^{\infty}
$$

where $\mathscr{C}^{\infty}$ denote the category of $C^{\infty}$ manifolds with border.

\subsection{The tangent groupoid.}

Definition 3.8 (Tangent groupoid). Let $\mathscr{G} \rightrightarrows \mathscr{G}^{(0)}$ be a Lie groupoid. The tangent groupoid associated to $\mathscr{G}$ is the groupoid that has $\mathscr{D}_{\mathscr{G}(0)}^{\mathscr{G}}$ as the set of arrows and $\mathscr{G}^{(0)} \times[0,1]$ as the units, with:

. $s^{T}(x, \eta, 0)=(x, 0)$ and $r^{T}(x, \eta, 0)=(x, 0)$ at $t=0$.

. $s^{T}(\gamma, t)=(s(\gamma), t)$ and $r^{T}(\gamma, t)=(r(\gamma), t)$ at $t \neq 0$.

- The product is given by $m^{T}((x, \eta, 0),(x, \xi, 0))=(x, \eta+\xi, 0)$ and $m^{T}((\gamma, t),(\beta, t))=(m(\gamma, \beta), t)$ if $t \neq 0$ and if $r(\beta)=s(\gamma)$.

- The unit map $u^{T}: \mathscr{G}^{(0)} \rightarrow \mathscr{G}^{T}$ is given by $u^{T}(x, 0)=(x, 0)$ and $u^{T}(x, t)=$ $(u(x), t)$ for $t \neq 0$.

We denote $\mathscr{G}^{T}:=\mathscr{D}_{\mathscr{G}(0)}^{\mathscr{G}}$.

As we have seen above $\mathscr{G}^{T}$ can be considered as a $C^{\infty}$ manifold with border. As a consequence of the functoriality of the DNC construction we can show that the tangent groupoid is in fact a Lie groupoid. Indeed, it is easy to check that if we identify in a canonical way $\mathscr{D}_{\mathscr{G}(0)}^{\mathscr{G}^{(2)}}$ with $\left(\mathscr{G}^{T}\right)^{(2)}$, then

$$
m^{T}=\mathscr{D}(m), s^{T}=\mathscr{D}(s), r^{T}=\mathscr{D}(r), u^{T}=\mathscr{D}(u)
$$


where we are considering the following pair morphisms:

$$
\begin{aligned}
m & :\left((\mathscr{G})^{(2)}, \mathscr{G}^{(0)}\right) \rightarrow\left(\mathscr{G}, \mathscr{G}^{(0)}\right), \\
s, r & :\left({ }^{(}, \mathscr{G}^{(0)}\right) \rightarrow\left(\mathscr{G}^{(0)}, \mathscr{G}^{(0)}\right), \\
u & :\left(\mathscr{G}^{(0)}, \mathscr{G}^{(0)}\right) \rightarrow\left({ }_{G}, \mathscr{G}^{(0)}\right) .
\end{aligned}
$$

Finally, if $\left\{\mu_{x}\right\}$ is a smooth Haar system on $\mathscr{G}$, then, setting

- $\mu_{(x, 0)}:=\mu_{x}$ at $\left(\mathscr{G}^{T}\right)_{(x, 0)}=T_{x} \mathscr{G}_{x}$ and

- $\mu_{(x, t)}:=t^{-q} \cdot \mu_{x}$ at $\left(\mathscr{G}^{T}\right)_{(x, t)}=\mathscr{G}_{x}$ for $t \neq 0$, where $q=\operatorname{dim} \mathscr{G}_{x}$,

one obtains a smooth Haar system for the Tangent groupoid (details may be found in 22]).

Examples 3.9. We finish this section with some interesting examples of groupoids and their tangent groupoids.

(i) The tangent groupoid of a group. Let $G$ be a Lie group considered as a Lie groupoid, $\mathscr{G}:=G \rightrightarrows\{e\}$. In this case the normal bundle to the inclusion $\{e\} \hookrightarrow G$ is of course identified with the Lie algebra of the Group. Hence, the tangent groupoid is a deformation of the group in its Lie algebra:

$$
\mathscr{G}^{T}=\mathfrak{g} \times\{0\} \bigsqcup G \times(0,1] .
$$

(ii) The tangent groupoid of a smooth vector bundle. Let $E \stackrel{p}{\rightarrow} X$ be a smooth vector bundle over a $C^{\infty}$ manifold $X$ (connexe). We can consider the Lie groupoid $E \rightrightarrows X$ induced by the vector structure of the fibers, i.e., $s(\xi)=p(\xi)=r(\xi)$ and the composition is given by the vector sum $\xi \circ \eta=\xi+\eta$. In this case the normal vector bundle associated to the zero section can be identified to $E$ itself. Hence, as a set the tangent groupoid is $E \times[0,1]$ but the $C^{\infty}$-structure at zero is given locally as in (3).

(iii) The tangent groupoid of a $C^{\infty}$ - manifold. Let $M$ a $C^{\infty}$-manifold. We can consider the product groupoid $\mathscr{G}_{M}:=M \times M \rightrightarrows M$. The tangent groupoid in this case takes the following form

$$
\mathscr{G}_{M}^{T}=T M \times\{0\} \sqcup M \times M \times(0,1] .
$$

This is called the tangent groupoid to $M$ and it was introduced by Connes for giving a very conceptual proof of the Atiyah-Singer index theorem (see 8$]$ and [10]).

\section{An algebra for the Tangent groupoid}

In this section we will show how to construct an algebra for the tangent groupoid which consist of $C^{\infty}$ functions that satisfy a rapid decay condition at zero while out of zero they satisfy a compact support condition. This algebra is the main construction in this work. 
4.1. Schwartz type spaces for Deformation to the normal cone manifolds. Our algebra for the Tangent groupoid will be a particular case of a construction associated to any deformation to the normal cone. We start by defining a space for DNCs associated to open subsets of $\mathbf{R}^{p} \times \mathbf{R}^{q}$.

Definition 4.1. Let $p, q \in \mathbf{N}$ and $U \subset \mathbf{R}^{p} \times \mathbf{R}^{q}$ an open subset, and let $V=$ $U \cap\left(\mathbf{R}^{p} \times\{0\}\right)$.

(1) Let $K \subset U \times[0,1]$ be a compact subset. We say that $K$ is a conic compact subset of $U \times[0,1]$ relative to $V$ if

$$
K_{0}=K \cap(U \times\{0\}) \subset V
$$

(2) Let $g \in C^{\infty}\left(\Omega_{V}^{U}\right)$. We say that $f$ has compact conic support $K$, if there exists a conic compact $K$ of $U \times[0,1]$ relative to $V$ such that if $t \neq 0$ and $(x, t \xi, t) \notin K$ then $g(x, \xi, t)=0$.

(3) We denote by $\mathscr{S}_{r, c}\left(\Omega_{V}^{U}\right)$ the set of functions $g \in C^{\infty}\left(\Omega_{V}^{U}\right)$ that have compact conic support and that satisfy the following condition:

$\left(s_{1}\right) \forall k, m \in \mathbf{N}, l \in \mathbf{N}^{p}$ and $\alpha \in \mathbf{N}^{q}$ it exists $C_{(k, m, l, \alpha)}>0$ such that

$$
\left(1+\|\xi\|^{2}\right)^{k}\left\|\partial_{x}^{l} \partial_{\xi}^{\alpha} \partial_{t}^{m} g(x, \xi, t)\right\| \leq C_{(k, m, l, \alpha)}
$$

Now, the spaces $\mathscr{S}_{r, c}\left(\Omega_{V}^{U}\right)$ are invariant under diffeomorphisms. More precisely if $F: U \rightarrow U^{\prime}$ is a $C^{\infty}$ diffeomorphism as in lemma 3.2 then we can prove the next result.

Proposition 4.2. Let $g \in \mathscr{S}_{r, c}\left(\Omega_{V^{\prime}}^{U^{\prime}}\right)$, then $\tilde{g}:=g \circ \tilde{F} \in \mathscr{S}_{r, c}\left(\Omega_{V}^{U}\right)$.

Proof. The first observation is that $\tilde{g} \in C^{\infty}\left(\Omega_{V}^{U}\right)$, thanks to lemma 3.2, Let us check that it has compact conic support. For that, let $K^{\prime} \subset U^{\prime} \times[0,1]$ the conic compact support of $g$. We let

$$
K=\left(F^{-1} \times i d_{[0,1]}\right) \subset U \times[0,1],
$$

which is a conic compact subset of $U \times[0,1]$ relative to $V$, and it is immediate by definition that $\tilde{g}(x, \xi, t)=0$ if $t \neq 0$ and $(x, t \cdot \xi, t) \notin K$, that is, $\tilde{g}$ has compact conic support $K$.

We now check the rapid decay property $\left(s_{1}\right)$ : For simplify the proof we first introduce some useful notation. Writing $F=\left(F_{1}, F_{2}\right)$ as in the lemma 3.2, we denote $F_{1}(x, \xi)=\left(A_{1}(x, \xi), \ldots, A_{p}(x, \xi)\right)$ and $F_{2}(x, \xi)=\left(B_{1}(x, \xi), \ldots, B_{q}(x, \xi)\right)$. We denote also $w=w(x, \xi, t)=\left(A_{1}(x, t \xi), \ldots, A_{p}(x, t \xi)\right)$ and $\eta=\eta(x, \xi, t)=$ $\left(\tilde{B}_{1}(x, \xi, t), \ldots, \tilde{B}_{q}(x, \xi, t)\right)$ where $\tilde{B}_{j}$ is also as above, i.e.,

$$
\tilde{B}_{j}(x, \xi, t)=\left\{\begin{array}{cc}
\frac{\partial B_{j}}{\partial \xi}(x, 0) \cdot \xi & \text { if } t=0 \\
\frac{1}{t} B_{j}(x, t \xi) & \text { if } t \neq 0
\end{array}\right.
$$


In particular by definition we have $\tilde{F}(x, \xi, t)=(w, \eta, t)$. We also write $z=(x, \xi, t)$ and $u=(\omega, \eta, t)$. Hence, what we would like is to find bounds for expressions of the following type

$$
\|\xi\|^{k}\left\|\partial_{z}^{\alpha} \tilde{g}(z)\right\|,
$$

for arbitrary $k \in \mathbf{N}$ and $\alpha \in \mathbf{N}^{p} \times \mathbf{N}^{q} \times \mathbf{N}$. A simple calculation shows that the derivates $\partial_{z}^{\alpha} \tilde{g}(z)$ are of the following form

$$
\partial_{z}^{\alpha} \tilde{g}(z)=\sum_{|\beta| \leq|\alpha|} P_{\beta}(z) \partial_{u}^{\beta} g(u)
$$

where $P_{\beta}(z)$ is a finite sum of products of the form

$$
\partial_{z}^{\gamma} \omega_{i}(z) \cdot \partial_{z}^{\delta} \eta_{j}(z)
$$

We are only interested in see what happens in the set $K_{\Omega}:=\{z=(x, \xi, t) \in \Omega$ : $(x, t \cdot \xi, t) \in K\}$ since out of this set we have that $g$ and all its derivates vanish $\left((x, t \xi, t) \in K\right.$ iff $\left.(w, t \eta, t) \in K^{\prime}\right)$. For a point $z=(x, \xi, t) \in K_{\Omega}$ we have that $(x, t \cdot \xi)$ is in a compact set and then it follows that the expressions

$$
\left\|\partial_{z}^{\gamma} \omega_{i}(z)\right\|
$$

are bounded in $K_{\Omega}$. For the expressions $\left\|\partial_{z}^{\delta} \eta_{j}(z)\right\|$, we proceed first by developing as in lemma 3.2. that is,

$$
\eta_{j}(x, \xi, t)=\left(\frac{\partial B_{j}}{\partial \xi}(x, 0) \cdot \xi+h^{j}(x, t \xi)\right) \cdot \xi
$$

Now, since we are only considering points in $K_{\Omega}$, it is immediate that we can find constants $C_{j}>0$ such that

$$
\left\|\partial_{z}^{\delta} \eta_{j}(z)\right\| \leq C_{j} \cdot\|\xi\|^{m_{\delta}} .
$$

In the same way (remember $F$ is a diffeomorphism) we can have constants $C_{i}>0$ such that

$$
\left\|\xi_{i}(\omega, \eta, t)\right\| \leq C_{i} \cdot\|\eta\| .
$$

Putting all together, and using the property $\left(s_{1}\right)$ for $g$, we get bounds $C>0$ such that

$$
\|\xi\|^{k}\left\|\partial_{z}^{\alpha} \tilde{g}(z)\right\| \leq C,
$$

and this concludes the proof.

Remark 4.3. We can resume the last invariance result as follows: If $(\mathscr{U}, \mathscr{V})$ is a $C^{\infty}$ pair diffeomorphic to $(U, V)$ with $U \subset E$, an open subset of a vector space $E$, and $V=U \cap E$, then $\mathscr{S}_{r, c}\left(\mathscr{D}_{\mathscr{Y}}^{\mathscr{U}}\right)$ is well defined and does not depend on the pair diffeomorphism.

With the last compatibility result in hand we are ready to give the main definition in this work. 
Definition 4.4. Let $g \in C^{\infty}\left(\mathscr{D}_{X}^{M}\right)$.

(a) We say that $g$ has compact conic support $K$, if there exists a compact subset $K \subset M \times[0,1]$ with $K_{0}:=K \cap(M \times\{0\}) \subset X$ (conic compact relative to $X)$ such that if $t \neq 0$ and $(m, t) \notin K$ then $g(m, t)=0$.

(b) We say that $g$ is rapidly decaying at zero if for every $(\mathscr{U}, \phi) X$-slice chart and for every $\chi \in C_{c}^{\infty}(\mathscr{U} \times[0,1])$, the map $g_{\chi} \in C^{\infty}\left(\Omega_{V}^{U}\right)$ given by

$$
g_{\chi}(x, \xi, t)=\left(g \circ \varphi^{-1}\right)(x, \xi, t) \cdot\left(\chi \circ p \circ \varphi^{-1}\right)(x, \xi, t)
$$

is in $\mathscr{S}_{r, c}\left(\Omega_{V}^{U}\right)$, where $p$ is the projection $p: \mathscr{D}_{X}^{M} \rightarrow M \times[0,1]$ given by $(x, \xi, 0) \mapsto(x, 0)$, and $(m, t) \mapsto(m, t)$ for $t \neq 0$.

Finally, we denote by $\mathscr{S}_{r, c}\left(\mathscr{D}_{X}^{M}\right)$ the set of functions $g \in C^{\infty}\left(\mathscr{D}_{X}^{M}\right)$ that are rapidly decaying at zero with compact conic support.

Remark 4.5. (a) By definition of $\mathscr{S}_{r, c}\left(\mathscr{D}_{X}^{M}\right)$ we see that $C_{c}^{\infty}\left(\mathscr{D}_{X}^{M}\right)$ is contained as a vector subspace.

(b) It is clear that $C_{c}^{\infty}(M \times(0,1])$ can be considered as a subspace of $\mathscr{S}_{r, c}\left(\mathscr{D}_{X}^{M}\right)$ by extending by zero the functions at $\mathscr{N}_{X}^{M}$.

Following the lines of the last remark we are going to precise a possible decomposition of our space $\mathscr{S}_{r, c}\left(\mathscr{D}_{X}^{M}\right)$ that will be very useful in the sequel. Let $\left\{\left(\mathscr{U}_{\alpha}, \phi_{\alpha}\right)\right\}_{\alpha \in \Delta}$ a family of $X$-slices covering $X$. Consider the open cover of $M \times[0,1]$ consisting in $\left\{\left(\mathscr{U}_{\alpha} \times[0,1], \phi_{\alpha}\right)\right\}_{\alpha \in \Delta}$ union with $M \times(0,1]$. We can take a partition of the unity subordinated to the last cover,

$$
\left\{\chi_{\alpha}, \lambda\right\}_{\alpha \in \Delta}
$$

That is, we have the following properties:

$$
\begin{aligned}
& \cdot 0 \leq \chi_{\alpha}, \lambda \leq 1 \\
& \cdot \operatorname{supp} \chi_{\alpha} \subset \mathscr{U}_{\alpha} \times[0,1] \text { and } \operatorname{supp} \lambda \subset M \times(0,1] . \\
& \cdot \sum_{\alpha} \chi_{\alpha}+\sum \lambda=1
\end{aligned}
$$

Let $f \in \mathscr{S}_{r, c}\left(\mathscr{D}_{X}^{M}\right)$, we denote

$$
f_{\alpha}:=\left.f\right|_{\mathscr{D}_{\mathscr{V}_{\alpha}}^{\mathscr{U}_{\alpha}}} \cdot\left(\chi_{\alpha} \circ p\right) \in C^{\infty}\left(\mathscr{D}_{\mathscr{V}_{\alpha}}^{\mathscr{U}_{\alpha}}\right)
$$

and

$$
f_{\lambda}:=\left.f\right|_{M \times(0,1]} \cdot(\lambda \circ p) \in C^{\infty}(M \times(0,1]),
$$

then we obtain the following decomposition:

$$
f=\sum_{\alpha} f_{\alpha}+f_{\lambda}
$$

Now, since $f$ is conic compactly supported we can suppose, without lost of generality, that 
$\cdot f_{\lambda} \in C_{c}^{\infty}(M \times(0,1])$, and

- that $\chi_{\alpha}$ is compactly supported in $\mathscr{U}_{\alpha} \times[0,1]$.

What we conclude of all this, is that we can decompose our space $\mathscr{S}_{r, c}\left(\mathscr{D}_{X}^{M}\right)$ as follows

$$
\mathscr{S}_{r, c}\left(\mathscr{D}_{X}^{M}\right)=\sum_{\alpha \in \Lambda} \mathscr{S}_{r, c}\left(\mathscr{D}_{\mathscr{V}_{\alpha}}^{\mathscr{U}_{\alpha}}\right)+C_{c}^{\infty}(M \times(0,1]) .
$$

As we mentioned in the introduction, we want to see the space $\mathscr{S}_{r, c}\left(\mathscr{D}_{X}^{M}\right)$ as a field of vector spaces over the interval $[0,1]$, where at zero we talked about Schwartz spaces. In our case we are interested in Schwartz functions on the vector bundle $\mathscr{N}_{X}^{M}$. Let us first recall the notion of the Schwartz space associated to a vector bundle.

Definition 4.6. Let $(E, p, X)$ be a smooth vector bundle over a $C^{\infty}$ manifold $X$. We define the Schwartz space $\mathscr{S}(E)$ as the set of $C^{\infty}$ functions $g \in C^{\infty}(E)$ such that $g$ is a Schwartz function at each fiber (uniformly) and $g$ has compact support in the direction of $X$, i.e., if there exists a compact subset $K \subset X$ such that $g\left(E_{x}\right)=0$ for $x \notin K$.

The vector space $\mathscr{S}(E)$ is an associative algebra with the product given as follows: for $f, g \in \mathscr{S}(E)$, we put

$$
(f * g)(\xi)=\int_{E_{p(\xi)}} f(\xi-\eta) g(\eta) d \mu_{p(\xi)}(\eta),
$$

where $\mu_{\xi}$ is a smooth Haar system of the Lie groupoid $E \rightrightarrows X$. A classical Fourier argument can be applied to show that the last algebra is isomorphic to $\left(\mathscr{S}\left(E^{*}\right), \cdot\right)$ (punctual product). In particular this implies that $K_{0}(\mathscr{S}(E)) \cong K^{0}\left(E^{*}\right)$.

In the case we are interested, we have a couple $(M, X)$ and a vector bundle associated to it, that is, the normal bundle over $X, \mathscr{N}_{X}^{M}$. The reason why we gave the last definition is because we get evaluation linear maps

$$
e_{0}: \mathscr{S}_{r, c}\left(\mathscr{D}_{X}^{M}\right) \rightarrow \mathscr{S}\left(\mathscr{N}_{X}^{M}\right),
$$

and

$$
e_{t}: \mathscr{S}_{r, c}\left(\mathscr{D}_{X}^{M}\right) \rightarrow C_{c}^{\infty}(M)
$$

for $t \neq 0$. Consequently, we have that the vector space $\mathscr{S}_{r, c}\left(\mathscr{D}_{X}^{M}\right)$ is a field of vector spaces over the closed interval $[0,1]$, which fibers spaces are: $\mathscr{S}\left(\mathscr{N}_{X}^{M}\right)$ at $t=0$ and $C_{c}^{\infty}(M)$ for $t \neq 0$.

Examples 4.7. Let us finish this subsection by giving the examples of spaces $\mathscr{S}_{r, c}\left(\mathscr{D}_{X}^{M}\right)$ corresponding to the DCN manifolds seen at 3.5 above.

1. For $X=\emptyset$, we have that $\mathscr{S}_{r, c}\left(\mathscr{D}_{\emptyset}^{M}\right) \cong C_{c}^{\infty}(M \times(0,1])$.

2. For $X \subset M$ an open subset we have that $\mathscr{S}_{r, c}\left(\mathscr{D}_{X}^{M}\right) \cong C_{c}^{\infty}(W)$ where $W \subset$ $M \times[0,1]$ is the open subset consisting of the union of $X \times[0,1]$ and $M \times(0,1]$. 
4.2. Schwartz type algebra for the Tangent groupoid. In this section we define an algebra structure on $\mathscr{S}_{r, c}\left(\mathscr{G}^{T}\right)$. We start by defining a function $m_{r, c}: \mathscr{S}_{r, c}\left(\mathscr{D}_{\mathscr{G}(0)}^{\mathscr{G}^{(2)}}\right) \rightarrow \mathscr{S}_{r, c}\left(\mathscr{D}_{\mathscr{G}(0)}^{\mathscr{G}}\right)$ by the following formulas:

For $F \in \mathscr{S}_{r, c}\left(\mathscr{D}_{\mathscr{G}(0)}^{\mathscr{G}^{(2)}}\right)$, we let

$$
m_{r, c}(F)(x, \xi, 0)=\int_{T_{x} \mathscr{G}_{x}} F(x, \xi-\eta, \eta, 0) d \mu_{x}(\eta)
$$

and

$$
m_{r, c}(F)(\gamma, t)=\int_{\mathscr{G}_{s(\gamma)}} F\left(\gamma \circ \delta^{-1}, \delta, t\right) t^{-q} d \mu_{s(\gamma)}(\delta)
$$

If we canonically identify $\mathscr{D}_{\mathscr{G}(0)}^{\mathscr{G}^{(2)}}$ with $\left(\mathscr{G}^{T}\right)^{(2)}$, the map above is nothing else that the integration along the fibers of $m^{T}:\left(\mathscr{G}^{T}\right)^{(2)} \rightarrow \mathscr{G}^{T}$. We have the following proposition:

Proposition 4.8. $m_{r, c}: \mathscr{S}_{r, c}\left(\left(\mathscr{G}^{T}\right)^{(2)}\right) \rightarrow \mathscr{S}_{r, c}\left(\mathscr{G}^{T}\right)$ is a well defined linear map.

The interesting part of the proposition is that the map is well defined since it will evidently be linear. Let us suppose for the moment that the last proposition is true. Under this assumption, we will define the product in $\mathscr{S}_{r, c}\left(\mathscr{G}^{T}\right)$.

Definition 4.9. Let $f, g \in \mathscr{S}_{r, c}\left(\mathscr{G}^{T}\right)$, we define a function $f * g$ in $\mathscr{G}^{T}$ by

$$
(f * g)(x, \xi, 0)=\int_{T_{x} \mathscr{G}_{x}} f(x, \xi-\eta, 0) g(x, \eta, 0) d \mu_{x}(\eta)
$$

and

$$
(f * g)(\gamma, t)=\int_{\mathscr{G}_{s(\gamma)}} f\left(\gamma \circ \delta^{-1}, t\right) g(\delta, t) t^{-q} d \mu_{s(\gamma)}(\delta)
$$

for $t \neq 0$.

We can enounce our main result.

Theorem 4.10. $*$ defines an associative product on $\mathscr{S}_{r, c}\left(\mathscr{G}^{T}\right)$.

Proof. Remember we are assuming for the moment the proposition 4.8. Let $f, g \in$ $\mathscr{S}_{r, c}\left(\mathscr{G}^{T}\right)$. We let $F:=(f, g)$ the function in $\left(\mathscr{G}^{T}\right)^{(2)}$ defined by:

$$
(f, g)(x, \xi, \eta, 0)=f(x, \xi, 0) \cdot g(x, \eta, 0)
$$

and

$$
(f, g)((\gamma, t),(\delta, t))=f(\gamma, t) \cdot g(\delta, t)
$$

for $t \neq 0$. Now, from the Leibnitz formula for the derivate of a product it is immediate that $(f, g) \in \mathscr{S}_{r, c}\left(\left(\mathscr{G}^{T}\right)^{(2)}\right)$. Finally, by definition we have that

$$
m_{r, c}((f, g))=f * g
$$

hence, thanks to proposition $4.8 f * g$ is a well defined element in $\mathscr{S}_{r, c}\left(\mathscr{G}^{T}\right)$. 
For the associativity of the product, let us remark that when one restrict the product to $C_{c}^{\infty}\left(\mathscr{G}^{T}\right)$, this coincides with the product classically considered on $C_{c}^{\infty}\left(\mathscr{G}^{T}\right)$ (which is associative, see for example [22]). The associativity for $\mathscr{S}_{r, c}\left(\mathscr{G}^{T}\right)$ is proved exactly in the same way that for $C_{c}^{\infty}\left(\mathscr{G}^{T}\right)$.

We have then to prove proposition 4.8. We are going to start locally. Let $U \in \mathbf{R}^{p} \times \mathbf{R}^{q} \times \mathbf{R}^{q}$ be an open set and $V=U \cap \mathbf{R}^{p} \times\{0\} \times\{0\}$. Let $P$ : $\mathbf{R}^{p} \times \mathbf{R}^{q} \times \mathbf{R}^{q} \rightarrow \mathbf{R}^{p} \times \mathbf{R}^{q}$ the canonical projection $(x, \eta, \xi) \mapsto(x, \eta)$. We set $U^{\prime}=P(U) \in \mathbf{R}^{p} \times \mathbf{R}^{q}$, then $U^{\prime}$ is also an open subset, $V \cong U^{\prime} \cap \mathbf{R}^{p} \times\{0\}$ and $\left.P\right|_{V}=I d_{V}$. We denote also by $P$ the restriction $P: U \rightarrow U^{\prime}$. We have as in lemma 3.2 a $C^{\infty}$ map $\tilde{P}: \Omega_{V}^{U} \rightarrow \Omega_{V}^{U^{\prime}}$, which in this case is explicitly written by

$$
\tilde{P}(x, \eta, \xi, t)=(x, \eta, t)
$$

We define $\tilde{P}_{r, c}: \mathscr{S}_{r, c}\left(\Omega_{V}^{U}\right) \rightarrow \mathscr{S}_{r, c}\left(\Omega_{V}^{U^{\prime}}\right)$ as follows

$$
\tilde{P}_{r, c}(F)(x, \eta, t)=\int_{\left\{\xi \in \mathbf{R}^{q}:(x, \eta, \xi, t) \in \Omega_{V}^{U}\right\}} F(x, \eta, \xi, t) d \xi .
$$

Let us prove the following lemma.

Lemma 4.11. $\tilde{P}_{r, c}: \mathscr{S}_{r, c}\left(\Omega_{V}^{U}\right) \rightarrow \mathscr{S}_{r, c}\left(\Omega_{V}^{U^{\prime}}\right)$ is well defined.

Proof. The first observation is that the integral in the definition of $\tilde{P}_{r, c}$ is always well defined. Indeed, we deduce it from the next two points:

. For $t=0, \xi \mapsto F(x, \eta, \xi, 0) \in \mathscr{S}\left(\mathbf{R}^{q}\right)$.

. For $t \neq 0, \xi \mapsto F(x, \eta, \xi, t) \in C_{c}^{\infty}\left(\mathbf{R}^{q}\right)$.

Once we can derivate under the integral symbol, we obtain that $\tilde{P}_{r, c}(F) \in C^{\infty}\left(\Omega_{V}^{U^{\prime}}\right)$. Then, we just have to show that $\tilde{P}_{r, c}(F)$ verifies the two conditions of the definition 4.1. For the first, if $K \subset U \times[0,1]$ is the compact conic support of $F$, then it is enough to put

$$
K^{\prime}=\left(P \times i d_{[0,1]}\right)(K)
$$

in order to obtain a conic compact subset of $U^{\prime} \times[0,1]$ relative to $V$ and to check that $K^{\prime}$ is the compact conic support of $\tilde{P}_{r, c}(F)$. Let us now verify the condition $\left(s_{1}\right)$. Let $k, m \in \mathbf{N}, l \in \mathbf{N}^{p}$ and $\beta \in \mathbf{N}^{q}$. We want to find $C_{(k, m, l, \beta)}>0$ such that

$$
\left(1+\|\eta\|^{2}\right)^{k}\left\|\partial_{x}^{l} \partial_{\eta}^{\beta} \partial_{t}^{m} \tilde{P}_{r, c}(F)(x, \eta, t)\right\| \leq C_{(k, m, l, \alpha)}
$$

For $k^{\prime} \geq k+\frac{q}{2}$ and $\alpha=(0, \beta) \in \mathbf{R}^{q} \times \mathbf{R}^{q}$ we have by hypothesis that it exists $C_{\left(k^{\prime}, m, l, \alpha\right)}^{\prime}>0$ such that

$$
\left\|\partial_{x}^{l} \partial_{\eta}^{\beta} \partial_{t}^{m} F(x, \eta, \xi, t)\right\| \leq C^{\prime} \frac{1}{\left(1+\|(\eta, \xi)\|^{2}\right)^{k^{\prime}}}
$$

Then, we also have that

$$
\left\|\partial_{x}^{l} \partial_{\eta}^{\beta} \partial_{t}^{m} \tilde{P}_{r, c}(F)(x, \eta, t)\right\| \leq C^{\prime} \int_{\left\{\xi \in \mathbf{R}^{q}:(x, \eta, \xi, t) \in \Omega_{V}^{U}\right\}} \frac{1}{\left(1+\|(\eta, \xi)\|^{2}\right)^{k^{\prime}}} d \xi
$$




$$
\leq C^{\prime} \frac{1}{\left(1+\|\eta\|^{2}\right)^{\frac{q}{2}-k^{\prime}}} \int_{\left\{\xi \in \mathbf{R}^{q}\right\}} \frac{1}{\left(1+\|\xi\|^{2}\right)^{k^{\prime}}} d \xi \leq C \frac{1}{\left(1+\|\eta\|^{2}\right)^{k}}
$$

with

$$
C=C^{\prime} \cdot \int_{\left\{\xi \in \mathbf{R}^{q}\right\}} \frac{1}{\left(1+\|\xi\|^{2}\right)^{k^{\prime}}} d \xi
$$

We can now give the proof of the proposition 4.8 .

Proof of 4.8. Let us first fix some notation. We suppose $\operatorname{dim} \mathscr{G}=p+q$ and $\operatorname{dim}$ $\mathscr{G}^{(0)}=p$, in particular this implies that $\operatorname{dim} \mathscr{G}^{(2)}=p+q+q$. Let $(\mathscr{U}, \phi)$ and $\left(\mathscr{U}^{\prime}, \phi^{\prime}\right)$ be $\mathscr{G}^{(0)}$-slices in $\mathscr{G}^{(2)}$ and $\mathscr{G}$ respectively such that the following diagram commutes

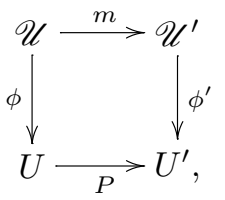

where $P: \mathbf{R}^{p} \times \mathbf{R}^{q} \times \mathbf{R}^{q} \rightarrow \mathbf{R}^{p} \times \mathbf{R}^{q}$ is the canonical projection (as above) and $P(U)=U^{\prime}$. This is possible since $m$ is a surjective submersion. Now, we apply the DNC construction to the diagram above to obtain, thanks to the functoriality of the construction, the following commutative diagram

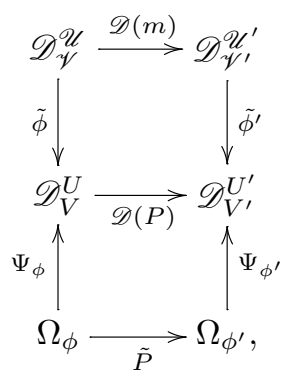

where $\Psi_{\phi}$ and $\tilde{\phi}$ are as in section 3 . Let $g \in \mathscr{S}_{r, c}\left(\mathscr{D}_{V}^{U}\right)$, we define

$$
P_{r, c}(g)(x, \eta, t)=\left\{\begin{array}{cl}
\int_{\mathbf{R}^{q}} g(x, \eta, \xi, 0) d \xi & \text { if } t=0 \\
\int_{\left\{\xi \in \mathbf{R}^{q}:(x, \eta, \xi, t) \in \Omega_{V}^{U}\right\}} g(x, \eta, \xi, t) t^{-q} d \xi & \text { if } t \neq 0
\end{array}\right.
$$

Then, from the last commutative diagram, we get that

$$
P_{r, c}(g)=\tilde{P}_{r, c}\left(g \circ \Psi_{\phi}\right) \circ\left(\Psi_{\phi^{\prime}}\right)^{-1},
$$

hence, thanks to 4.11. we can conclude that we have a well defined linear map

$$
P_{r, c}: \mathscr{S}_{r, c}\left(\mathscr{D}_{V}^{U}\right) \rightarrow \mathscr{S}_{r, c}\left(\mathscr{D}_{V^{\prime}}^{U^{\prime}}\right) \text {. }
$$


We now use the proposition 4.2 to write

$$
\mathscr{S}_{r, c}\left(\mathscr{D}_{\mathscr{V}}^{\mathscr{U}}\right)=\left\{h \in C^{\infty}\left(\mathscr{D}_{\mathscr{V}}^{\mathscr{U}}\right): h \circ \tilde{\phi}^{-1} \in \mathscr{S}_{r, c}\left(\mathscr{D}_{V}^{U}\right)\right\},
$$

and so for $h \in \mathscr{S}_{r, c}\left(\mathscr{D}_{\mathscr{V}}^{\mathscr{U}}\right)$, we see that

$$
P_{r, c}\left(h \circ \tilde{\phi}^{-1}\right) \circ \tilde{\phi}^{\prime} \in \mathscr{S}_{r, c}\left(\mathscr{D}_{\mathscr{V}^{\prime}}^{\mathscr{U}^{\prime}}\right) .
$$

We use again the last commutative diagram to see that

$$
m_{r, c}(h)=P_{r, c}\left(h \circ \tilde{\phi}^{-1}\right) \circ \tilde{\phi}^{\prime} .
$$

We then have a well defined linear map

$$
m_{r, c}: \mathscr{S}_{r, c}\left(\mathscr{D}_{\mathscr{V}}^{\mathscr{U}}\right) \rightarrow \mathscr{S}_{r, c}\left(\mathscr{D}_{\mathscr{V}^{\prime}}^{\mathscr{U}^{\prime}}\right)
$$

To pass to the global case we only have to use the decomposition of $\mathscr{S}_{r, c}\left(\mathscr{D}_{\mathscr{G}(0)}^{\mathscr{G}^{(2)}}\right)$ and of $\mathscr{S}_{r, c}\left(\mathscr{D}_{\mathscr{G}(0)}^{\mathscr{G}}\right)$ as in (5), and of course the invariance under diffeomorphisms (proposition 4.2).

Let us recall that we have well defined evaluation morphisms as in (7) and (8). In the case of a the tangent groupoid they are by definition morphisms of algebras. Hence, the algebra $\mathscr{S}_{r, c}\left(\mathscr{G}^{T}\right)$ is field of algebras over the closed interval $[0,1]$, with associated fiber algebras,

$$
\begin{gathered}
\mathscr{S}(A \mathscr{G}), \text { at } t=0, \text { and } \\
C_{c}^{\infty}(\mathscr{G}) \text { for } t \neq 0 .
\end{gathered}
$$

It is very interesting to see what this means in the examples given in 3.9 .

\section{Further developements}

Let $\mathscr{G} \rightrightarrows \mathscr{G}^{(0)}$ be a Lie groupoid. In index theory for Lie groupoids the tangent groupoid has been used to define the analytic index associated to the group, as a morphism $K^{0}\left(A^{*} \mathscr{G}\right) \rightarrow K_{0}\left(C_{r}^{*}(\mathscr{G})\right)$ (see [19]) or as a $K K$-element in $K K\left(C_{0}\left(A^{*} \mathscr{G}\right), C_{r}^{*}(\mathscr{G})\right)$ (see [13]), this can be done because one has the following short exact sequence of $C^{*}$-algebras

$$
0 \rightarrow C_{r}^{*}(\mathscr{G} \times(0,1]) \longrightarrow C_{r}^{*}\left(\mathscr{G}^{T}\right) \stackrel{e_{0}}{\longrightarrow} C_{0}\left(A^{*} \mathscr{G}\right) \longrightarrow 0,
$$

and because of the fact that the $K$-groups of the algebra $C_{r}^{*}(\mathscr{G} \times(0,1])$ vanish (homotopy invariance). The index defined at the $C^{*}$-level has proven to be very useful (see for example [9]) but extracting numerical invariants from it, with the existent tools, is very difficult. In non commutative geometry, and also in classical geometry, the tools for obtain more explicit invariants are more developed for the 'smooth objects'; in our case this means the convolution algebra $C_{c}^{\infty}(\mathscr{G})$, where we 
can for example apply Chern-Weil-Connes theory. Hence, in some way, the indices defined in $K_{0}\left(C_{c}^{\infty}(\mathscr{G})\right)$ are more refined objects and for some cases it would be preferable to work with them. Unfortunately this indices are not good enough, since for example they are not homotopy invariants; in [8] Alain Connes discusses this and also other reasons why it is not enough to keep with the $C_{c}^{\infty}$-indices. The main reason to construct the algebra $\mathscr{S}_{r, c}\left(\mathscr{G}^{T}\right)$ is that it gives an intermediate way between the $C_{c}^{\infty}$-level and the $C^{*}$-level and will allow us in [5] to define another analytic index morphism associated to the groupoid, with the advantage that this index will take values in a group that allows to do pairings with cyclic cocycles and in general to apply Chern-Connes theory to it. The way we are going to define our index is by obtaining first a short exact sequence analogue to (9), that is, a sequence of the following kind

$$
\left.0 \rightarrow J \longrightarrow \mathscr{S}_{r, c}\left(\mathscr{G}^{T}\right) \stackrel{e_{0}}{\longrightarrow} \mathscr{S}\left(A^{*} \mathscr{G}\right)\right) \longrightarrow 0 .
$$

The problem here will be that we do not dispose of the advantages of the $K$-theory for $C^{*}$-algebras, since the algebras we are considering are not of this type (we do not have for example homotopy invariance).

\section{References}

[1] Aastrup, J., Melo, S.T., Monthubert, B. Schrohe, E. Boutet de Monvel's Calculus and Groupoids I. Preprint arxiv:math.KT/0611336

[2] Aastrup, J., Nest, R. and Schrohe, E. A continuous field of $C^{*}$-algebras and the tangent groupoid for manifolds with boundary. J. Funct. Anal. 237 (2006), no.2, 482506.

[3] Brown, L., Green, P., Rieffel, M. Stable isomorphism and stable Morita equivalence for $C^{*}$-algebras. Pacific Journal of Math. 71 (1977), no.2, 349-363.

[4] Carinena, J., Clemente-Gallardo, J., Follana, E., Gracia-Bondia, J.M., Rivero, A., Varilly, J.C. Connes' tangent groupoid and strict quantization. J. Geom. Phys. 32 (1999), no.2, 79-96.

[5] Carrillo, P. An analytic index for Lie groupoids. Preprint arxiv:math.KT/0612455

[6] Connes, A. Sur la théorie non commutative de l'intégration. Lecture notes in Math. 725, Springer-Berlin (1979), 19-143.

[7] Connes, A. Noncommutative differential geometry I.H.E.S. no. 62, (1985), 257-360.

[8] Connes, A. Non commutative geometry. Academic Press,Inc,San Diego, CA (1994).

[9] Connes, A. and Skandalis, G. The longitudinal index theorem for foliations. In Publ.Res.Inst.Math 6 (1984), 1139-1183.

[10] Debord, C., Lescure, J.M., Nistor, V. Groupoids and an index theorem for conical pseudomanifolds. Preprint arxiv:math.OA/0609438.

[11] Dixmier, J. Les $C^{*}$-algèbres et leurs représentations. Cahiers Scientifiques, Fasc. XXIX Gauthier-Villars 83 Cie, Paris (1964).

[12] Haefliger, A. Groupoïdes d'holonomie et classifiants. Astérisque Transversal structure of foliations (1982), no. 116, 70-97. 
[13] Hilsum, M. and Skandalis, G. Morphismes $K$-orientés d'espaces de feuilles et fonctorialité en théorie de Kasparov (d'après une conjecture d'A.Connes) Ann.Sci.École Norm.Sup. (4) 20 (1987), no. 3, 325-390.

[14] Landsman, N.P. Quantization and the tangent groupoid. Operator algebras and mathematical physics (2003), 251-265.

[15] Lauter, R., Monthubert, B., Nistor, V. Pseudodifferential analysis on continuous family groupoids. Doc. Math 5 (2000), 625-655.

[16] Melrose, R. The Atiyah-Patodi-Singer index theorem. Research notes in Mathematics 4 AK Peters, Ltd., Wellesley, MA, (1993).

[17] Moerdijk, I. Orbifolds as groupoids: an introduction. Contemp. Math., 310, Amer. Math. Soc., Providence, RI, Orbifolds in mathematics and physics (Madison, WI, 2001), 205-222.

[18] Monthubert, B. Groupoids and Pseudodifferential calculus on manifolds with corners. Journal of Functional Analysis 199 (2003), 243-286.

[19] Monthubert, B. and Pierrot, F. Indice analytique et groupoïdes de Lie. C.R.Acad.Scien.Paris 325 (1997) no.2, 193-198.

[20] Moroinau, S. Adiabatic limits of eta and zeta functions of elliptic operators. Math. Z. 246 (2004), no. 3, 441-471.

[21] Nistor, V., Weinstein, A. and Xu, P. Pseudodifferential operators on differential groupoids. Pacific J. Math 189 (1999), no.1, 117-152.

[22] Paterson, A. Groupoids and inverse semigroups, and their operator algebras. Progress in Mathematics, 170 Birkhäuser Boston, Inc., Boston, MA, (1999).

[23] Renault, J. A groupoid approach to $C^{*}$-algebras. Lecture notes in Math. 793, Springer, Berlin, (1980).

[24] Winkelnkemper. The graph of a foliation. Annals of global analysis and geometry 1 (1983), no.3, 51-75.

Paulo Carrillo Rouse

Projet d'algèbres d'opérateurs

Université de Paris 7

175, rue de Chevaleret

Paris, France

E-mail: carrillo@math.jussieu.fr 